\title{
Direct simulation of phase delay effects on induced-charge electro-osmosis under large ac electric fields
}

\author{
Hideyuki Sugioka* \\ Frontier Research Center, Canon Inc., 30-2, Shimomaruko 3-chome, Ohta-ku, Tokyo 146-8501, Japan \\ and Department of Mechanical Systems Engineering, Shinshu University, 4-17-1 Wakasato, Nagano 380-8553, Japan
}

(Received 8 May 2016; revised manuscript received 9 July 2016; published 19 August 2016)

\begin{abstract}
The standard theory of induced-charge electro-osmosis (ICEO) often overpredicts experimental values of ICEO velocities. Using a nonsteady direct multiphysics simulation technique based on the coupled PoissonNernst-Planck and Stokes equations for an electrolyte around a conductive cylinder subject to an ac electric field, we find that a phase delay effect concerning an ion response provides a fundamental mechanism for electrokinetic suppression. A surprising aspect of our findings is that the phase delay effect occurs even at much lower frequencies (e.g., $50 \mathrm{~Hz}$ ) than the generally believed charging frequency of an electric double layer (typically, $1 \mathrm{kHz}$ ) and it can decrease the electrokinetic velocities in one to several orders. In addition, we find that the phase delay effect may also cause a change in the electrokinetic flow directions (i.e., flow reversal) depending on the geometrical conditions. We believe that our findings move toward a more complete understanding of complex experimental nonlinear electrokinetic phenomena.
\end{abstract}

DOI: 10.1103/PhysRevE.94.022609

Induced-charge electro-osmosis (ICEO) [1], which includes the concept of ac electro-osmosis (ACEO) [2,3], is a nonlinear electrokinetic phenomenon resulting from the interaction between a tangential electric field and a diffused charge that forms an electric double layer with an induced charge on a conductive material; it is expected to be a key phenomenon that provides various innovative microfluidic devices, such as a pump [4], mixer [5], valve [6], etc. [1,7], since ICEO provides large flow velocities $(\sim \mathrm{mm} / \mathrm{s})$ with low applied voltages $(\sim \mathrm{V})$. In fact, Urbanski et al. [4] experimentally reported a much faster pump $(\sim 0.4 \mathrm{~mm} / \mathrm{s}$ at $3 \mathrm{~V})$ using ACEO with a step electrode structure than ordinary electrokinetic pumps (e.g., $\sim 0.1 \mathrm{~mm} / \mathrm{s}$ at $\sim \mathrm{kV}$ ). However, the standard theory using a thin electric double layer approximation [1] often overpredicts the measured flow velocities by a factor of 10 or more, and sometimes even the flow direction is opposite that of the predicted direction [8,9]. Moreover, it is known that ICEO flow ceases at high ion concentrations $(>10 \mathrm{mM})$ [8]. Thus, several models have been proposed to explain the above problems from the beginning. For example, early on, Gomayunov et al. [10] observed a reverse flow phenomenon for conductive particles, and they conjectured that it is due to a Faradaic reaction. Pascall and Squires successfully showed that contamination of the surface provides a natural mechanism for suppression of ICEO velocities by systematically measuring ICEO flow velocities over controllably contaminated planar electrodes [11]. Storey et al. showed that crowding of ions due to steric effects and a related decrease in surface capacitance could cause the experimentally observed flow reversal of planar ACEO pumps [12], and Bazant et al. pointed out that ion crowding also can cause the $10 \mathrm{mM}$ limit problem and the suppression of ICEO velocities at large voltages $(>25 \mathrm{mV})$ [8]. Nevertheless, even the simplest ICEO flow around a

*hsugioka@shinshu-u.ac.jp conductive cylinder (or particle) has not been explained completely and challenging problems still remain, e.g., even now we cannot explain completely the complex transition between the standard and anomalous vortex flows caused by changing the applied frequencies and voltages, although we pointed out that the phase delay of ions can cause a significant decrease in ICEO flows and ICEO flow reversal by using the one-dimensional (1D) numerical analysis of Poisson-Nernst-Planck (PNP) equations [13].

Recently, two-dimensional (2D) direct calculation methods that calculate ICEO flows directly based on the governing equations without using a thin double layer approximation have been developed by several groups [14-16]. For example, Gregersen et al. [14] developed a 2D steady direct calculation method and analyzed steady ICEO flows above a planar electrode of the geometry corresponding to Pascall and Squires's experiments. Davidson et al. [15] reported a chaotic ICEO flow around a conductive cylinder by using a 2D nonsteady direct calculation method. Further, Sugioka [16] performed a 2D stable direct analysis for a dc step response problem of ICEO flows around a conductive cylinder at large voltages by developing a stable multiphysics calculation method that assures the conservation of ion numbers completely, and showed that a shortage of ions (not crowding of ions) at large voltages can cause a decrease of ICEO flow velocities in the presence of dc electric fields.

However, ICEO flows around a conductive cylinder in the presence of large ac electric fields have yet to be analyzed by the $2 \mathrm{D}$ nonsteady direct calculation method. Therefore, in this paper, we focus on the $2 \mathrm{D}$ direct simulation around a conductive cylinder under a large ac electric field and fundamentally clarify the suppression problem of ICEO flows. In particular, by using the $2 \mathrm{D}$ nonsteady direct simulation that considers the Poisson-Nernst-Planck (PNP) equations with the Stokes equation, we will show that the phase delay effect on ions under ac electric fields is a natural fundamental mechanism to cause significant suppression and reversal of ICEO flows. 

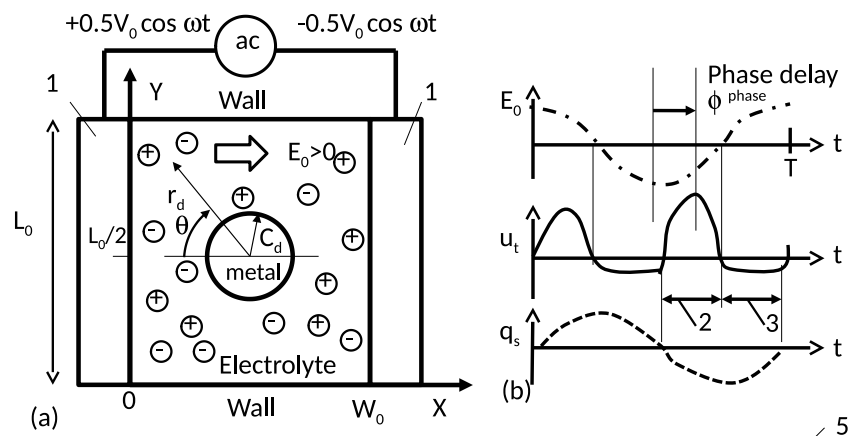

(c)
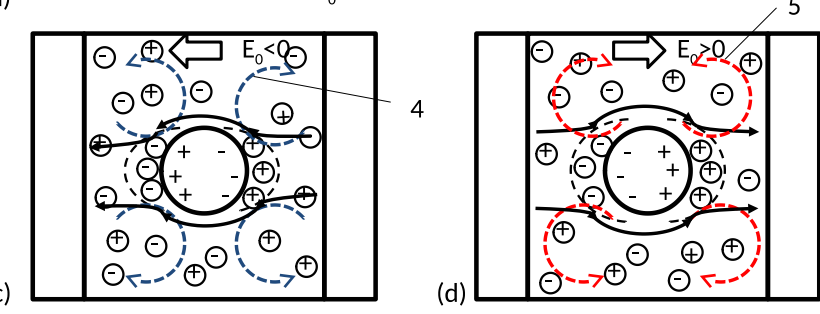

FIG. 1. Considered 2D model system of our direct simulation under ac electric fields and suppression mechanism due to a phase delay of an ion response. Here, $V_{0}$ is the peak ac applied voltage and $W_{0}(=100 \mu \mathrm{m})$ is the distance between the electrodes. (a) System. (b) Time chart. (c) Standard vortex flow. (d) Anomalous vortex flow. 1: electrodes, 2: period of standard vortex flow, 3: period of anomalous vortex flow, 4: standard vortex flow, 5: anomalous vortex flow.

Figure 1 shows a considered geometry of the 2D direct simulation [16] under ac electric fields and suppression mechanism due to the phase delay of an ion response. As shown in Fig. 1(a), we analyze an ICEO flow around a metal cylinder of radius $c_{d}$ in an electrolyte between parallel electrodes of distance $W_{0}$ (typically, $100 \mu \mathrm{m}$ ) under ac applied voltages of amplitude $V_{0}$ and frequency $f_{0}=\frac{1}{T_{0}}$ [i.e., $V_{\mathrm{ac}}=$ $\left.V_{0} \cos \left(2 \pi f_{0} t\right)\right]$. Here, the electrolyte is typically water of $p \mathrm{H}=7$ and thus we can assume that the Debye screening length $\lambda_{D}$ is ideally $1 \mu \mathrm{m}$; the dielectric permittivity $\varepsilon$ and viscosity $\mu$ are $80 \varepsilon_{0}$ and $1 \mathrm{mPas}$, respectively, where $\varepsilon_{0}$ is the vacuum permittivity. As illustrated in Fig. 1(b), the second peak of the extrema value of tangential velocities $u_{t}^{\text {extrema }}$ at $\theta=45^{\circ}$ generally appears at $t=\frac{T_{0}}{2}+t_{\phi}$ and thus we can define the phase delay of $u_{t}$ as $\phi^{\text {phase }}=2 \pi \frac{t_{\phi}}{T_{0}} \operatorname{rad}$. Note that the phase delay of $u_{t}$ is due to the phase delay of an ion response to applied ac voltages, and the phase delay of ions is represented by an accumulated charge $q_{s}$ in the electric double layer. It is defined as $q_{s}=\int_{c_{d}}^{c_{d}+d_{\mathrm{ex}}} \rho_{e} d r$, where $d_{\mathrm{ex}}$ is the distance that provides the extrema value of $u_{t}, \rho_{e}\left(=C_{+}-C_{-}\right)$is the charge density, $C_{+}\left(C_{-}\right)$is the concentration of positive (negative) ions, and $r$ is a radial axis. Since the signs of $E_{0}$ and $q_{s}$ provide the sign of $u_{t}$, the value of $u_{t}$ sometimes becomes negative in the presence of the phase delay of ions. In other words, not only the standard vortex flow of Fig. 1(c) but also the anomalous vortex flow [of Fig. 1(d)] can be naturally observed at least theoretically if there is a phase delay of ions, although the time averaging value of $u_{t}$ should also be negative to observe ICEO flow reversal experimentally. Further, the method of the $2 \mathrm{D}$ direct simulation we have done is the same as that of our previous study [16], except that here we consider an ac electric field instead of a dc electric field; namely, one can find detailed explanations on our direct simulation method in Ref. [16], however, for the convenience of readers, we briefly explain the method here. That is, the $2 \mathrm{D}$ direct simulation using the Poisson equation $\left(\varepsilon \nabla^{2} \Phi+\rho_{e}=0\right)$, Nernst-Planck equation $\left[\frac{\partial C_{ \pm}}{\partial t}+\nabla \cdot\left[-D\left(\nabla C_{ \pm} \pm \frac{z e}{k T} C_{ \pm} \nabla \Phi\right)+C_{ \pm} \boldsymbol{u}\right]=0\right]$, and Stokes equation $\left(-\nabla p+\mu \nabla^{2} \boldsymbol{u}-\rho_{e} \nabla \Phi=\mathbf{0}, \nabla \cdot \boldsymbol{u}=0\right)$ is performed by using the finite element method (FEM) with the finite volume method (FVM). Here, $\Phi$ is the potential, $t$ is time, $\boldsymbol{u}$ is the flow velocity, $p$ is pressure, $\mu(\sim 1 \mathrm{mPas})$ is the viscosity, $D\left(\sim 10^{-9} \mathrm{~m}^{2} / \mathrm{s}\right)$ is the ion diffusivity, $k$ is the Boltzmann constant, $T$ is the temperature, and $z e$ is the ion charge.

Figure 2 shows the calculation results of the phase delay effects under the condition that $c_{d} / W_{0}=0.1, W_{0}=$ $100 \mu \mathrm{m}, \lambda_{D}=1 \mu \mathrm{m}, V_{0}=1 \mathrm{~V}$, and $f_{0}=200 \mathrm{~Hz}$. As shown in Fig. 2(a), by applying a voltage [dashed line: $\left.V_{\mathrm{ac}}=V_{0} \cos \left(2 \pi f_{0} t\right)\right], \quad q_{s}$ approximately behaves as $q_{s} \sim$ $\frac{q_{s}^{\max }-q_{s}^{\min }}{2} \sin \left(2 \pi f_{0} t\right)$ and $u_{t}$ approximately behaves as $u_{t} \sim$ $\left[\frac{u_{t}^{\max }-u_{t}^{\min }}{2} \sin \left(4 \pi f_{0} t\right)-\frac{u_{t}^{\max }+u_{t}^{\min }}{2}\right]$, although each wave form is complex in detail, where $q_{s}^{\max }$ and $q_{s}^{\min }\left(u_{t}^{\max }\right.$ and $\left.u_{t}^{\min }\right)$ are the maximum and minimum values of $q_{s}\left(u_{t}\right)$. Thus, the phase delay of $u_{t}$ is $\sim \frac{\pi}{4}$ in this argument, although $\phi^{\text {phase }}=28.8^{\circ}$ at $f_{0}=200 \mathrm{~Hz}$ in detail. This rough estimation is simple but it shows a surprising fact that the phase delay of $u_{t}$ around a circular cylinder is intrinsically not negligible and it can dramatically suppress the average ICEO flow velocities. In our understanding, this fact has not been recognized well in the field of ICEO so far, although we pointed out the phase delay effect in our previous papers $[9,13]$. Please note that the wave forms of $u_{t}$ during the first and second periods are almost the same and thus we define the phase delay value from the second peak value of $u_{t}$.

Further, another surprising aspect of Fig. 2(a) is that the flow direction easily becomes opposite to that of the standard theory under the existence of the phase delay of ions; e.g., you can find the negative region of $u_{t}$ during $3.8 \leqslant t \leqslant 4.6 \mathrm{~ms}$ in Fig. 2(a). More specifically, Figs. 2(b)-2(d) show the dependence of $E_{t}$, $\rho_{e}$, and $u_{t}$, respectively, on $d_{0}$ at $t=2.9,3.4,3.9$, and $4.3 \mathrm{~ms}$. Namely, even though the sign of the tangential electric fields $E_{t}$ changes from negative to positive at $t=\frac{T_{0}}{4}=3.75 \mathrm{~ms}$, as shown in Fig. 2(b), the sign of the charge density $\rho_{e}$ remains negative, as shown in Fig. 2(c); thus, the sign of $u_{t}$ becomes positive at $t=2.9$ and $3.4 \mathrm{~ms}$, while it becomes negative at $t=3.9$ and $4.3 \mathrm{~ms}$, as shown in Fig. 2(d). Therefore, we can find a standard vortex flow at $t=2.9 \mathrm{~ms}$, as shown in Fig. 2(e), while we can find an anomalous vortex flow at $t=4.3 \mathrm{~ms}$, as shown in Fig. 2(e). Note that we use a different magnified factor to draw the flow vectors of Figs. 2(e) and 2(d) to clarify the reverse flow problem.

Figure 3 shows the dependence on $f_{0}$ under the condition that $c_{d} / W_{0}=0.06-0.1, V_{0}=1 \mathrm{~V}, W_{0}=100 \mu \mathrm{m}$, and $\lambda_{d}=1 \mu \mathrm{m}$ (i.e., $p \mathrm{H}=7$ as an ideal limit). As shown in Fig. 3(a), the average tangential flow velocity (defined as $\left.u_{t}^{\text {ave }}=\frac{1}{T_{0}} \int_{0}^{T_{0}} u_{t} d t\right)$ decreases as $f_{0}$ increase, however, the most surprising aspect of Fig. 3(a) is that the typical ICEO flow velocity around the conductive cylinder is at most $45 \mu \mathrm{m} / \mathrm{s}$ under ac electric fields, and this theoretical prediction is consistent with the experimental results measured by many researchers $[8-10,13,17]$. Thus, the phase delay effects under ac electric fields is a natural mechanism of the suppression 


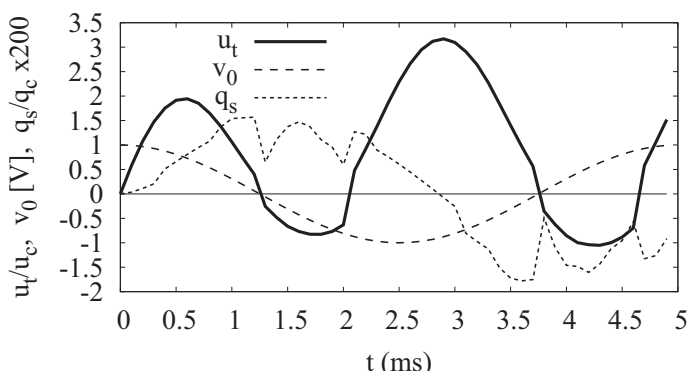

(a)

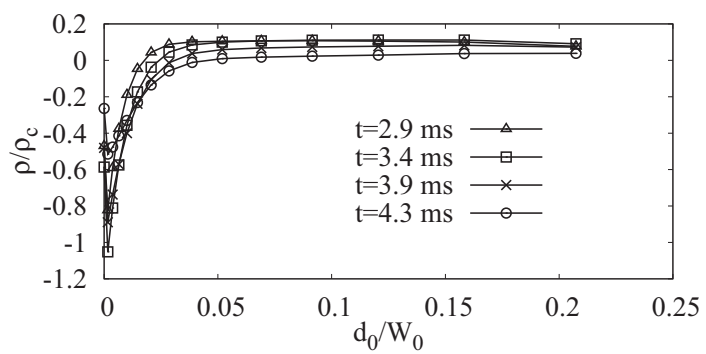

(c)

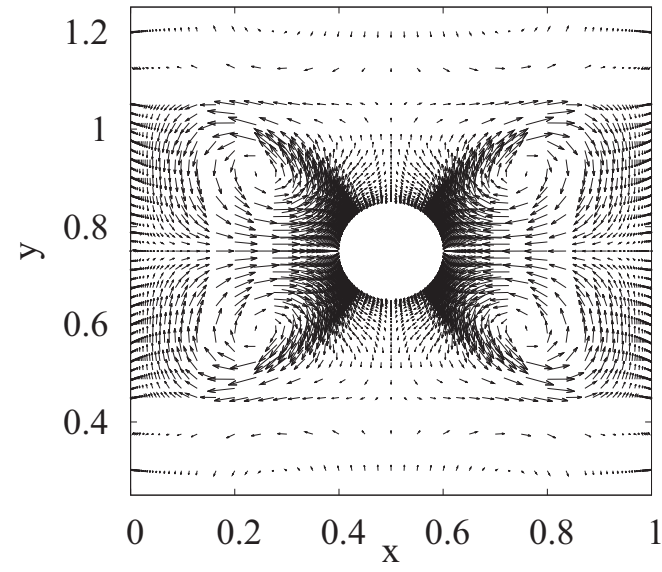

(e)

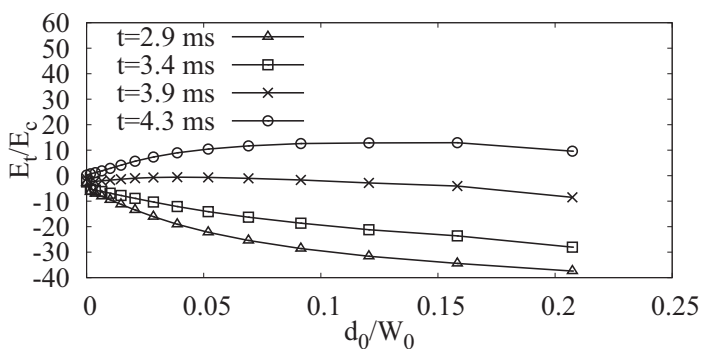

(b)

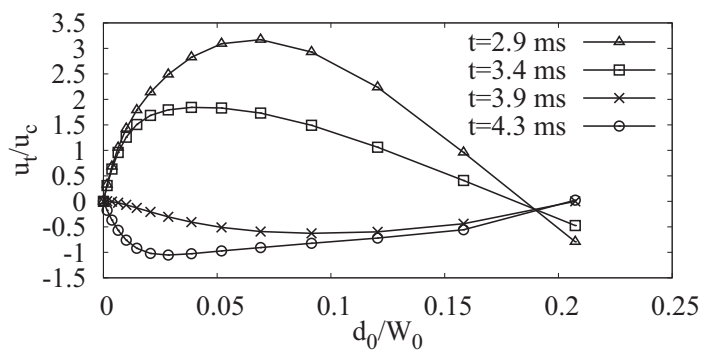

(d)

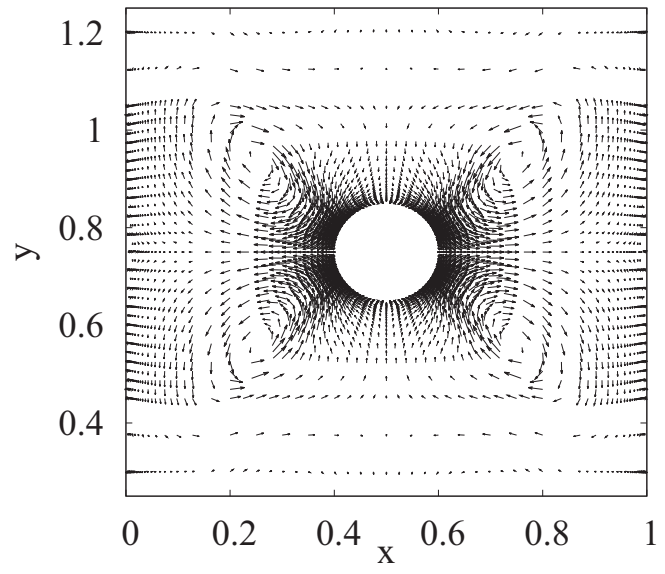

(f)

FIG. 2. Phase delay effects $\left(200 \mathrm{~Hz}, W_{0}=100 \mu \mathrm{m}\right.$, and $\left.V_{0}=1 \mathrm{~V}\right)$. Here, $d_{0}\left(=r-c_{d}\right)$ is a distance from the surface of the conductive cylinder, $c_{d} / W_{0}=0.1, W_{0}=100 \mu \mathrm{m}, D=10^{-9} \mathrm{~m}^{2} / \mathrm{s}, \lambda_{D}=1 \mu \mathrm{m}, u_{c}=D / w_{0}=10 \mu \mathrm{m} / \mathrm{s}, q_{c}=e z C_{0} V_{0}$, and $p \mathrm{H}=7$. (a) Dependence of $u_{t}^{\text {extrema }}, v_{0}$, and $q_{s}$ on $t\left(\theta=45^{\circ}\right)$. (b) $E_{t}$ vs $d_{0}\left(\theta=45^{\circ}\right)$. (c) $\rho$ vs $d_{0}\left(\theta=45^{\circ}\right)$. (d) $u_{t}$ vs $d_{0}\left(\theta=45^{\circ}\right)$. (e) Standard vortex flow field $(t=2.9 \mathrm{~ms}$, factor $=0.01)$. (f) Anomalous vortex flow field due to phase delay $(t=4.3 \mathrm{~ms}$, factor $=0.02)$.

of ICEO flow velocities measured in experiments. Please note that since the represented ICEO flow velocity is that $u_{0}^{\text {iceo }}=\varepsilon c_{d} E_{0}^{2} / \mu=708 \mu \mathrm{m} / \mathrm{s}$ at $c_{d}=10 \mu \mathrm{m}$ and $V_{0}=1 \mathrm{~V}$ $\left(E_{0}=V_{0} / W_{0}=10 \mathrm{kV} / \mathrm{m}\right)$, the typical value of $u_{t} / u_{0}^{\text {iceo }}$ under ac electric fields is $\sim 0.06$ at most; this situation is very different from that of the direct simulation of a dc step response of ICEO [16] since it shows that the value of $u_{t} / u_{0}^{\text {iceo }}$ under dc electric fields is approximately 0.5 at the same condition. Figure $3(\mathrm{~b})$ shows the dependence of $\phi^{\text {phase }}$ on $f_{0}$. Obviously, non-negligible values of $\phi^{\text {phase }}$ ranging from $26^{\circ}$ to $40^{\circ}$ in Fig. 2(b) are the main reason for the suppression of ICEO flow velocity. However, since $\phi^{\text {phase }}$ decreases in the range $50 \leqslant f_{0} \leqslant 150 \mathrm{~Hz}$, the reason why $u_{t}$ decreases as $f_{0}$ increases is different from the phase delay effects. Instead, it is explained by the monotonous decrease of $q_{s}$ as $f_{0}$ increases, as shown in Figs. 3(c) and 3(d). As the result, $u_{t}$ almost monotonously decreases as $f_{0}$ increases, while $\phi^{\text {phase }}$ decreases (increases) at $f_{0} \leqslant 150 \mathrm{~Hz}\left(f_{0} \geqslant 150 \mathrm{~Hz}\right)$, as shown in Figs. 3(e) and 3(f).

In addition, in Fig. 2(b), we can find that there exists a clear resonance phenomenon of $\phi^{\text {phase }}$ at $f_{0} \simeq 200 \mathrm{~Hz}$. We consider that the background physics of this phenomenon is the same as the well-known low-frequency dielectric dispersion (or alpha dispersion) [18] which is associated with the relaxation of the double layer surrounding the colloidal particles [18] since the resonant frequency in Fig. 3(b) is generalized as $f_{0}^{\text {reso }}\left(W_{0}^{2} / D\right) \simeq 2000$ on the basis of a nondimensional argument, i.e., our calculation results such as $u_{t}^{\text {ave }} W_{0} / D$ and $\phi^{\text {phase }}$ are the function of $F_{0}=k T C_{0}\left(W_{0}^{2} / D\right) \mu=\frac{\varepsilon}{2 \mu D}\left(\frac{k T}{e z}\right)^{2}\left(\frac{W_{0}}{\lambda_{D}}\right)^{2}$, $c_{d} / W_{0}$, and $e z V_{0} / k T$ [16], where $C_{0}$ is the ion concentration of the bulk. Namely, if $c_{d} / W_{0}$ and $e z V_{0} / k T$ are constant, $f_{0}^{\text {reso }} \sim$ $1 /\left(W_{0}^{2} / D\right)$, and the behavior of $f_{0}^{\text {reso }}$ of $\phi^{\text {phase }}$ corresponds to that of the $\alpha$ dispersion (i.e., $f_{\alpha} \sim c_{d}^{2} / D$ [18]) to some extent 


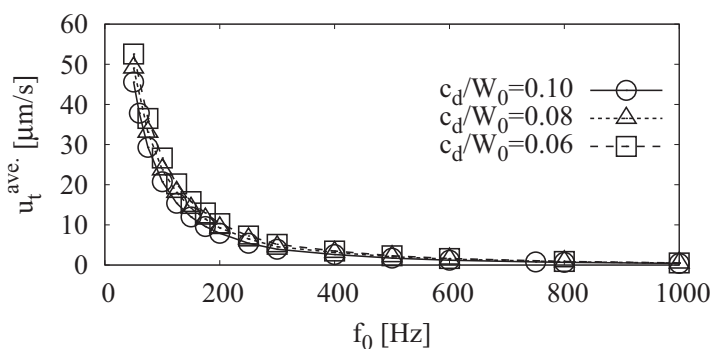

(a)

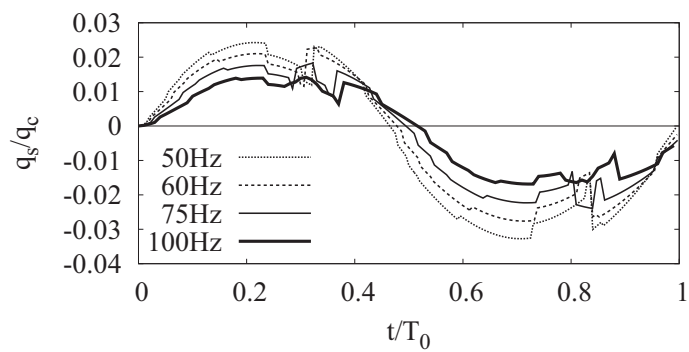

(c)

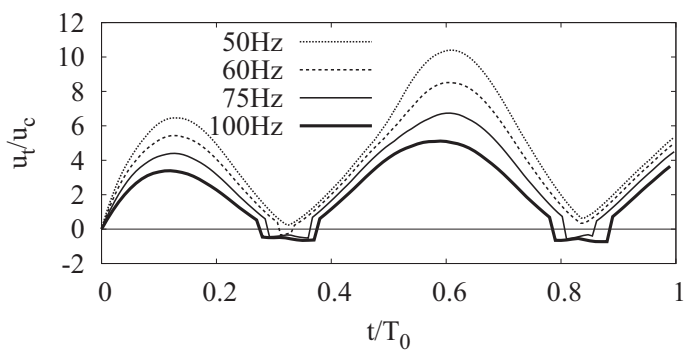

(e)

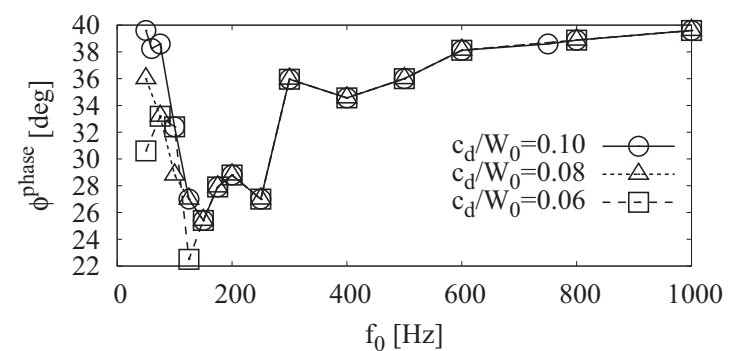

(b)

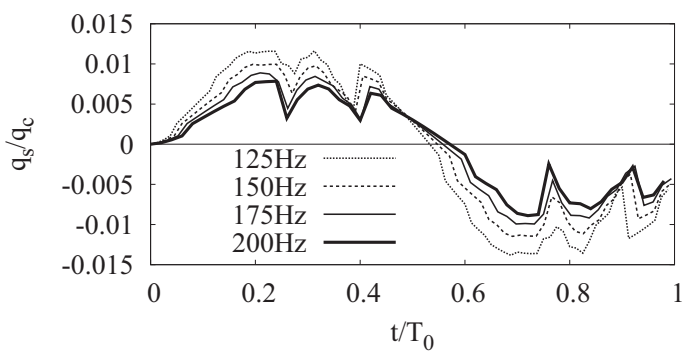

(d)

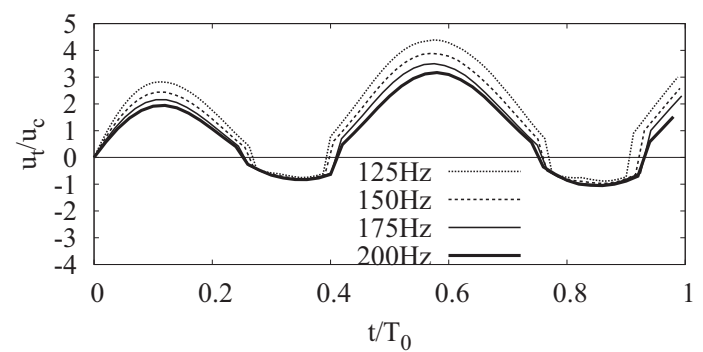

(f)

FIG. 3. Dependence of $u_{t}^{\text {ave }}$ and $\phi^{\text {phase }}$ on $f_{0}$. Here, $\theta=45^{\circ}, V_{0}=1 \mathrm{~V}, W_{0}=100 \mu \mathrm{m}, D=10^{-9} \mathrm{~m}^{2} / \mathrm{s}, \lambda_{D}=1 \mu \mathrm{m}, u_{0}^{\text {iceo }}=708 \mu \mathrm{m} / \mathrm{s}$, $q_{c}=e z C_{0} W_{0}$, and $p \mathrm{H}=7$. (a) Dependence of $u_{t}^{\text {ave }}$ on $f_{0}$. (b) Dependence of $\phi^{\text {phase }}$ on $f_{0}$. (c) Dependence of $q_{s}$ on $t\left(50 \leqslant f_{0} \leqslant 100 \mathrm{~Hz}\right.$; $\left.c_{d} / W_{0}=0.1\right)$. (d) Dependence of $q_{s}$ on $t\left(125 \leqslant f_{0} \leqslant 200 \mathrm{~Hz} ; c_{d} / W_{0}=0.1\right)$. (e) Dependence of $u_{t}$ on $t\left(50 \leqslant f_{0} \leqslant 100 \mathrm{~Hz} ; c_{d} / W_{0}=0.1\right)$. (f) Dependence of $u_{t}$ on $t\left(125 \leqslant f_{0} \leqslant 200 \mathrm{~Hz} ; c_{d} / W_{0}=0.1\right)$.

(at least in a specific experimental condition). However, as shown in Fig. 3(b), the resonance frequency does not directly depend on $c_{d} / W_{0}$; it is strongly dependent on $C_{0}$ or $\lambda_{D}$ if $W_{0}$ and $V_{0}$ are constant, since it changes the nondimensional value $F_{0}$. These phenomena are also known as the $\alpha$ dispersion due to a so-called volume diffusion mechanism related to the concentration polarization around a particle [18], whereas the $\alpha$ dispersion due to the surface diffusion mechanism is strongly characterized by $f_{\alpha} \sim c_{d}^{2} / D$ [18].

Figure 4 shows the dependence on $V_{0}$ at $f_{0}=50,100$, and $200 \mathrm{~Hz}$ under the condition that $c_{d} / W_{0}=0.1, W_{0}=100 \mu \mathrm{m}$, and $\lambda_{D}=1 \mu \mathrm{m}$. As shown in Figs. 4(a) and $4(\mathrm{~b}), u_{t}^{\text {ave }} / u_{0}^{\text {iceo }}$ and $\phi^{\text {phase }}$ almost do not change at $f_{0}=200 \mathrm{~Hz}$ as $V_{0}$ increases, whereas they change significantly at $f_{0}=50$ and $100 \mathrm{~Hz}$. Here, by considering the resonance curve in Fig. 3(b), we can explain the invariance of $\phi^{\text {delay }}$ on $V_{0}$ at $f_{0}=200 \mathrm{~Hz}$, i.e., around the resonance frequency $\left(f_{0}^{\text {reso }}=200 \mathrm{~Hz}\right)$, the phase delay change due to the small parameter change must be small because of the characteristic of the stable point. Further, as shown in Figs. 4(c) and 4(d), we can observe the shift of the peak value of $u_{t}$ and $q_{s}$ as $V_{0}$ increases. As a whole, the simulated complex behavior of $u_{t}$ on $f_{0}$ and $V_{0}$ agrees well with that in the experimental observation in Ref. [13], and the complex behavior of $u_{t}$ on $f_{0}$ and $V_{0}$ can be explained naturally by the phase delay effects, although we may need to compare the simulation results with the experimental results in detail in the future. For example, we may need to consider the steric limit in the direct simulation method as discussed in Ref. [7] (Sec. 5.4), although it is unlikely to exceed a steric limit for the dilute solution $\left(C_{0} \leqslant 10 \mathrm{mM}\right)$. Further, as a first step, we assume that the thickness of the Stern layer is zero for simplicity; however, the finite thickness of the Stern layer may amplify the complexity on $V_{0}$ and $f_{0}$, although it is beyond the scope of this paper.

Figure 5 shows the dependence on $W_{0}$ at $f_{0}=100$ and $200 \mathrm{~Hz}$ under the condition that $c_{d} / W_{0}=0.1$ (depicted by squares and circles) and under the condition that $c_{d}=10 \mu \mathrm{m}$ (depicted by crosses and triangles). Here, $V_{0}=1 \mathrm{~V}$ and $\lambda_{D}=$ $1 \mu \mathrm{m}$. As shown in Figs. 5(a) and 5(b), under the condition that $c_{d} / W_{0}=0.1, u_{t}^{\text {ave }}$ decreases monotonously at $f_{0}=100$ and $200 \mathrm{~Hz}$ as $W_{0}$ increases, although the dependence of $\phi^{\text {delay }}$ on $W_{0}$ has the peak values of $\phi^{\text {phase }}=38.7^{\circ}$ and $43.3^{\circ}$ at $W_{0}=50$ and $75 \mu \mathrm{m}$ for $f_{0}=100$ and $200 \mathrm{~Hz}$, respectively. Note that the decreasing of $u_{t}^{\text {ave }}$ with increased $W_{0}$ results from the decreasing of electric field $E_{0}=V_{0} / W_{0}$. Under the condition that $c_{d}=10 \mu \mathrm{m}$, the values of $u_{t}^{\text {ave }}$ and $\phi^{\text {phase }}$ are almost the 


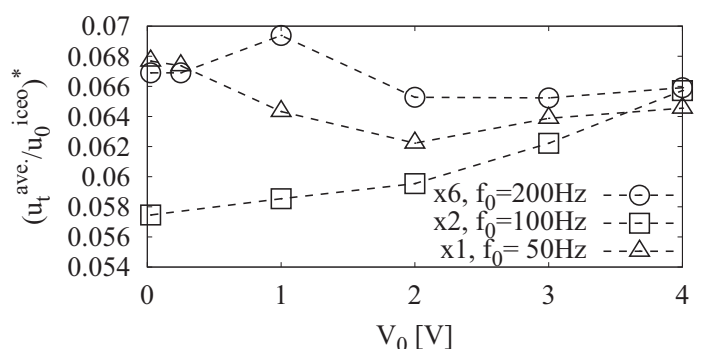

(a)

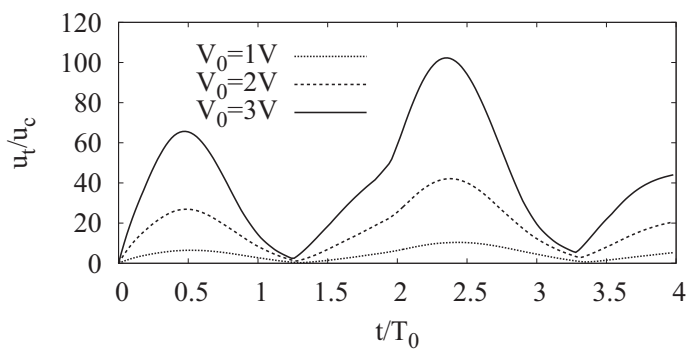

(c)

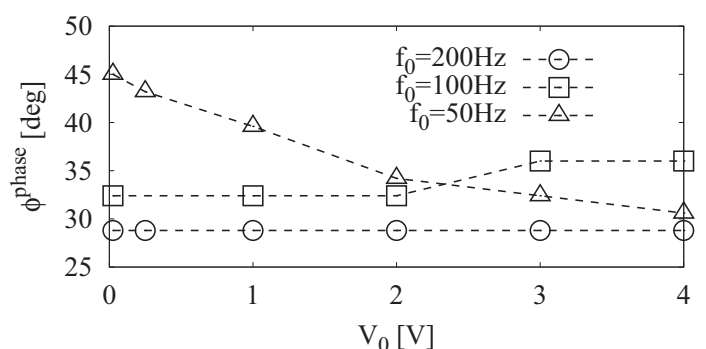

(b)

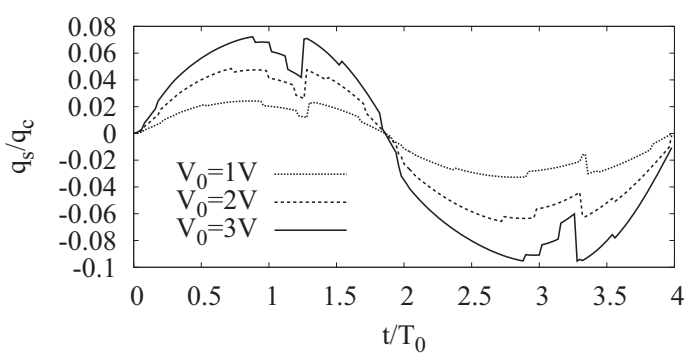

(d)

FIG. 4. Dependence of $u_{t}^{\text {ave }}$ and $\phi^{\text {phase }}$ on $V_{0}$. Here, $c_{d} / W_{0}=0.1, \theta=45^{\circ}, W_{0}=100 \mu \mathrm{m}, v_{c}=25 \mathrm{mV}, c_{0}^{\text {iceo }}=\epsilon c E_{0}^{2} / \mu, \mu=1 \mathrm{mPa} \mathrm{s}$, $f_{0}=200 \mathrm{~Hz}, W_{0}=100 \mu \mathrm{m}, D=10^{-9} \mathrm{~m}^{2} / \mathrm{s}, \lambda_{D}=1 \mu \mathrm{m}, q_{c}=e z C_{0} W_{0}$, and $p \mathrm{H}=7$. In (a), $u_{t}^{\text {ave }} / u_{0}^{\text {iceo }}$ is magnified by 1,2 , and 6 for $f_{0}=50,100$, and $200 \mathrm{~Hz}$, respectively, for the comparison of the tendency. (a) Dependence of $u_{t}^{\text {ave }}$ on $V_{0}$. (b) Dependence of $\phi^{\text {phase }}$ on $V_{0}$. (c) Dependence of $u_{t}$ on $t\left(f_{0}=50 \mathrm{~Hz}\right)$. (d) Dependence of $q_{s}$ on $t$.

same as those of $c_{d} / W_{0}=0.1$ at $W_{0} \geqslant 100 \mu \mathrm{m}$, although we can find the difference at $W_{0}<100 \mu \mathrm{m}$. Obviously, the difference between the two conditions at $W_{0}<100 \mu \mathrm{m}$ is owing to the boundary effect of the electrode, and the similarity of $u_{t}^{\text {ave }}$ and $\phi^{\text {phase }}$ at $W_{0} \geqslant 100 \mu \mathrm{m}$ means that the phase delay effect does not depend strongly on $c_{d}$ itself. This is because the simulated phenomenon belongs to the $\alpha$ dispersion due to a volume diffusion mechanism [18], as mentioned before. Furthermore, at high frequencies (e.g., $\left.f_{0}=3.2 \mathrm{kHz}\right), u_{t}^{\text {ave }}$ becomes nearly zero or shows slightly negative values, whereas $\phi^{\text {phase }}$ shows large values around $45^{\circ}$, e.g., $u_{t}^{\text {ave }}=-1.03 \mu \mathrm{m} / \mathrm{s}, \phi^{\text {phase }}=50.4^{\circ}$ at $W_{0}=25 \mu \mathrm{m}$ and $f_{0}=3.2 \mathrm{kHz}$, as shown in Figs. 5(a) and 5(b). Namely, in Figs. 5(c) and 5(d), the second peak positions of $u_{t}$ and the lines

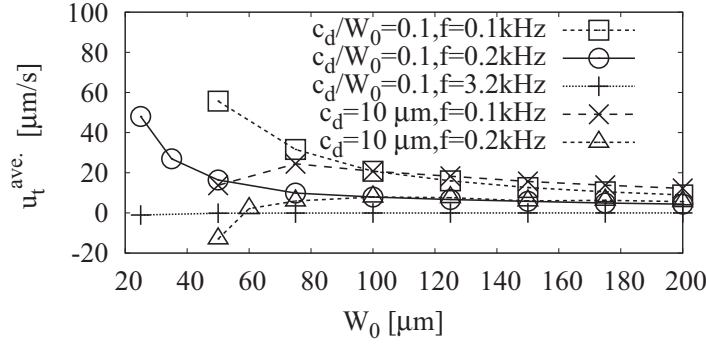

(a)

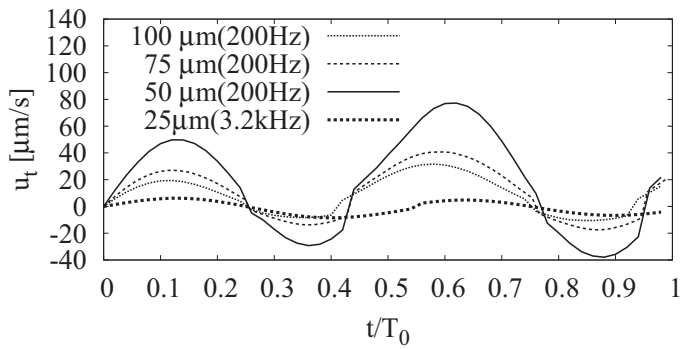

(c)

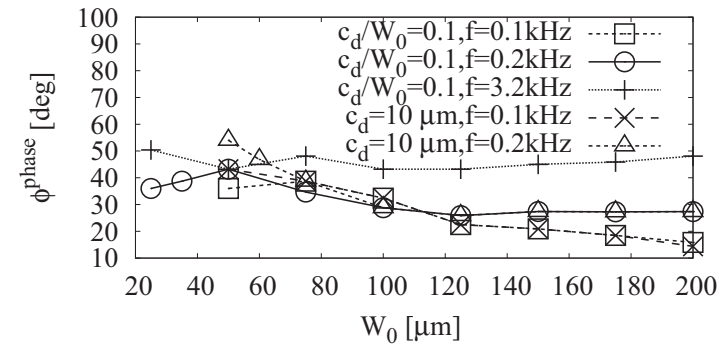

(b)

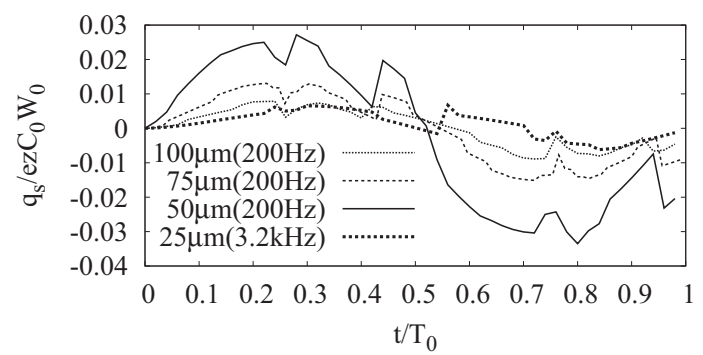

(d)

FIG. 5. Dependence of $u_{t}^{\text {ave }}$ and $\phi^{\text {phase }}$ on $W_{0}$. As $W_{0}$ increases, $\phi^{\text {phase }}$ decreases, thus, $u_{t} / u_{0}^{\text {iceo }}$ increases as $W_{0}$ increases. Here, $\theta=45^{\circ}$, $c_{0}^{\text {iceo }}=\epsilon c E_{0}^{2} / \mu, \mu=1 \mathrm{mPa} \mathrm{s}, V_{0}=1 \mathrm{~V}, f_{0}=200 \mathrm{~Hz}, D=10^{-9} \mathrm{~m}^{2} / \mathrm{s}, \lambda_{D}=1 \mu \mathrm{m}, q_{c}=e z C_{0} W_{0}, u_{c}=D / w_{0}=10 \mu \mathrm{m} / \mathrm{s}$, and $p \mathrm{H}=7$. (a) Dependence of $u_{t}^{\text {ave }}$ on $W_{0}$. (b) Dependence of $\phi^{\text {phase }}$ on $W_{0}$. (c) Dependence of $u_{t}^{\text {ave }}$ on $t\left(c_{d} / W_{0}=0.1\right)$. (d) Dependence of $q_{s}$ on $t$ $\left(c_{d} / W_{0}=0.1\right)$ 
of $q_{s}$ around $t / T_{0}=0.6$ are shifted to the left as $W_{0}$ increases, and the amplitudes of $u_{t}$ and $q_{s}$ become small at $f_{0}=3.2 \mathrm{kHz}$. Note that, in this case, the positive and negative flows of $u_{t}$ are almost balanced but show a slightly negative value. That is, we can find that the flow reversal of ICEO due to the phase delay of ions is observed in typical ICEO geometries and it also seriously suppresses the ICEO flow velocity. For example, since $u_{0}^{\text {iceo }}=2.83 \mathrm{~mm} / \mathrm{s}$ at $V_{0}=1 \mathrm{~V}$ and $W_{0}=25 \mu \mathrm{m}$, the correction factor $\Lambda_{\text {eff }}$ to the standard theory [1] is written as $\Lambda_{\text {eff }}=u_{t} / u_{0}^{\text {iceo }}=-0.00036$ in this case. This is a surprising prediction but an ordinary observed phenomenon by many researchers [8-10,13]; e.g., we experimentally reported that $\Lambda_{\text {eff }}=-0.0025$ for the ICEO flow velocities around a stacking structure of elliptic cylinders [9] and $\Lambda_{\text {eff }}=-0.0055$ for the ICEO flow velocities in the zigzag structure of circular cylinders [13]. Therefore, the phase delay of ions is also a natural mechanism of the flow reversal of ICEO, i.e., both the suppression and reversal of ICEO velocities first can be understood integratedly as the phase delay effect.

Our direct simulations clearly show that to consider the phase delay problem should be the first priority in future ICEO device design strategies since it is an intrinsic and fundamental problem for ideally clean and flat surfaces. In other words, the effects of contamination [11] and surface roughness [19] can be avoided by using a suitable fabrication process. Further, the problems of ion crowding [8] and shortage [16] are probably hidden by the phase delay effects since both phenomena are probably not growing at the existence of the phase delay effect. Of course, both phenomena may become important after the phase delay problem; however, in our opinion, the problems are not very serious since ion crowding is intrinsically a problem for dense solutions $\left(C_{0} \geqslant 10 \mathrm{mM}\right)$ [7] and ion shortage suppresses the ICEO velocity just by the factor of 0.5 or so [16]. Thus, the design strategy on the phase delay problem becomes the first priority. Furthermore, in the field of colloid science, the charging time $\tau_{D}$ and cutoff frequency $f_{D}$ for a colloidal particle is usually represented by $\tau_{D}=1 / f_{D}=\lambda_{D}^{2} / D$, although the charging time for a parallel electrode problem is considered to be $\tau_{R C}=L \lambda_{D} / D$, where $2 L$ is a gap distance of the parallel electrode [20]. Namely, the values of $\tau_{D}$ and $f_{D}$ are $1 \mathrm{~ms}$ and $1 \mathrm{kHz}$, respectively, under the condition that $D=10^{-9} \mathrm{~m}^{2} / \mathrm{s}$ and $\lambda_{D}=1 \mu \mathrm{m} / \mathrm{s}$, although the value of $f_{D}$ is usually larger than $10 \mathrm{kHz}$ since $\lambda_{D}$ is usually smaller than $100 \mathrm{~nm}$ at $C_{0} \geqslant 10^{-5} \mathrm{M}$. In fact, the peak forward and backward velocities of the ACEO pump having a step structure [4] are observed approximately at 10 and $100 \mathrm{kHz}$, respectively. Thus, our results are surprising since they show that the phase delay effects appear even at much lower frequencies (e.g., $50 \mathrm{~Hz}$ ) than the generally believed charging frequency (i.e., $1 \mathrm{kHz}$ ). At the same time, our findings make us understand various experimental reports on ICEO flow velocities without considering other extremely large effects [8-10,13]. For example, the trend of $\left|u_{t}^{\text {ave }}\right|$ decreasing with increased frequency is approximately consistent with experimental observations in Refs. [10,13,17]. Namely, experimental flow velocities of ICEO are explained well without considering extremely large correction factors (e.g., an extremely thick contamination or condensed layer, extremely large surface roughness, etc.) [8]. Please note that the time averaging value of the maximum flow velocity [e.g., in Fig. 2(d)] predicted from the simulation is assumed to be corresponding to the experimental maximum flow velocity around the metal cylinder or particle, as a first approximation. In addition, the ICEO devices are largely classified into two types, i.e., an ACEO-type device using two planar electrodes connected to a power source and a so-called ICEO-type device (in the narrow sense) that uses a conductive material in an electric field; our results suggest that the ACEO-type devices are better than the ICEO-type devices to avoid the phase delay problem since the ACEO pump using a step structure works well at high frequencies (e.g., $1 \mathrm{kKHz}$ ) in the experiment [4], as mentioned before, although the ICEO-type devices also might be improved for a specific frequency by adjusting the phase conditions. Further, the phase delay effect might be prevented for the planar electrode in the direction of the electric field [11]. However, to find the structure that prevents the phase delay effects is the beyond the scope of this paper, and we would like to explore the problem in the future.

We consider that the resonance phenomenon in Fig. 3(b) belongs to the so-called $\alpha$ dispersion phenomenon due to a volume diffusion mechanism related to the concentration mechanism. In particular, as a first step, we assume that the thickness of the effective relaxation region around the cylinder in the ac electric field $d^{\text {eff }}$ is similar to that in the dc electric field in Ref. [16], i.e., $d^{\text {eff }} \simeq 4 \lambda_{D}$. Thus, our system can be modeled by the $R C$ electrical circuit consisting of the resistance of the relaxation region $R^{\text {relax }}=\frac{\lambda_{D}^{2} d_{\text {eff }}}{\varepsilon D}$ and the capacitance of the electric double layer $C_{D}=\epsilon / \lambda_{D}$, and the relaxation time is represented by $\tau^{\text {relax }}=R^{\text {relax }} C_{D}=\frac{\lambda_{D} d^{\text {eff }}}{D} \simeq$ $4 \frac{\lambda_{D}^{2}}{D}$, e.g., $\tau^{\text {relax }}=4 \mathrm{~ms}$ for $D=10^{-9} \mathrm{~m}^{2} / \mathrm{s}$ and $\lambda_{D}=1 \mu \mathrm{m}$ [20]. Of course, it is considered as a complex impedance circuit consisting of $1 / j \omega C_{D}$ and $R^{\text {relax }}$ for the applied voltage $V_{a} \simeq \frac{c_{d}}{W_{0}} V_{0} e^{j \omega t}$, where $\frac{c_{d}}{W_{0}}$ is the order of the applied voltage for the particle. Further, the tangential electric field at the outside edge of the electric double layer is assumed to be $E_{t} \sim \frac{V_{0}}{W_{0}} e^{j \omega t}=E_{0} e^{j \omega t}$ and the zeta potential of the electric double layer $\zeta$ is obtained as the voltage drop of the capacitor, i.e., $\zeta=\frac{\frac{1}{j \omega C_{D}}}{R^{\text {relax }}+\frac{1}{j \omega C_{D}}} \frac{c_{d} V_{0}}{W_{0}} e^{j \omega t}=\frac{\omega \tau^{\text {relax }}}{\sqrt{1+\left(\omega \tau^{\text {relax }}\right)^{2}}} \frac{c_{d} V_{0}}{W_{0}} e^{j(\omega t-\phi)}$, where $\phi=\tan ^{-1}\left(\omega C_{D} R^{\text {relax }}\right)$. Namely, from the HelmholtzSmoluchowski formula, we obtain $u_{t} \simeq \frac{\varepsilon \operatorname{Re}(\zeta) \operatorname{Re}\left(E_{t}\right)}{\mu} \sim u_{t 0} g(t)$, where $g(t)=\cos (\omega t-\phi) \cos \omega t$ and $u_{t 0}=\frac{\varepsilon c_{d} E_{0}^{2}}{\mu} \frac{\omega \tau^{\text {relax }}}{\sqrt{1+\left(\omega \tau^{\text {relax }}\right)^{2}}}$. Here, $\operatorname{Re}(\zeta)$ and $\operatorname{Re}\left(E_{t}\right)$ mean the real part of $\zeta$ and $E_{t}$. By considering the extrema value condition that $d g / d t=$ 0 , we obtain the second extrema time that provides the extrema value of $u_{t}$ is $t^{\text {extrema }}=\left(0.5+\frac{\phi}{4 \pi}\right) T_{0}$. Thus, from our definition of $\phi^{\text {phase }}$, we obtain $\phi^{\text {phase,theory }} \simeq 2 \pi \frac{\phi}{4 \pi}=$ $\frac{\phi}{2}=\frac{1}{2} \tan ^{-1}\left(\omega C_{D} R^{\text {relax }}\right)$, e.g., $\phi \simeq 90^{\circ}$ and $\phi^{\text {phase }} \simeq 45^{\circ}$ at $1 / \omega C_{D} \ll R^{\text {relax }}$, whereas $\phi \simeq 45^{\circ}$ and $\phi^{\text {phase }} \simeq 22.5^{\circ}$ at $1 / \omega C_{D} \sim R^{\text {relax }}$. This is the mechanism of how phase delay occurs, and it successfully explains the large phase delay and our numerical results such as the dependence of $\phi^{\text {phase }}$ on frequency. Note that from the above discussion, we can consider that the phase delay does not change with the azimuthal angle, as a first step; thus, our numerical analysis at $\theta=45^{\circ}$ that provides the ICEO represented flow velocity $u_{0}$ is justified. Further, since $g(t)=\cos \phi \cos ^{2} \omega t-\frac{\sin \phi}{2} \sin 2 \omega t$, 
we obtain the time averaging phase term $\bar{g}=\frac{1}{T_{0}} \int_{0}^{T_{0}} g(t) d t=$ $\frac{\cos \phi}{2}$, e.g., $\bar{g}=0.35-0$ at $\phi=45^{\circ}-90^{\circ}$. This is the mechanism of how the phase delay effect suppresses the flow velocity, and it successfully explains the large suppression of $u_{t}^{\text {ave }}$ and our numerical results, such as the dependence of $u_{t}^{\text {ave }}$ on frequency.

To summarize, by 2D direct simulation, we have shown that a phase delay of an ion response to ac electric fields provides a natural mechanism for the significant suppression of ICEO flow velocities by a factor of 10 or more, and sometimes it may cause a reversal of ICEO flow directions depending on the geometrical conditions. We believe that our findings contribute to design strategies on innovative ICEO and ACEO devices.

This work was partially supported by JSPS KAKENHI Grant Numbers JP16K05650.
[1] M. Z. Bazant and T. M. Squires, Phys. Rev. Lett. 92, 066101 (2004).

[2] A. Ramos, H. Morgan, N. G. Green, and A. Castellanos, J. Colloid Interface Sci. 217, 420 (1999).

[3] A. Ramos, A. Gonzalez, A. Castellanos, N. G. Green, and H. Morgan, Phys. Rev. E 67, 056302 (2003).

[4] J. P. Urbanski, T. Thorsen, J. A. Levian, and M. Z. Bazant, Appl. Phys. Lett. 89, 143508 (2006).

[5] C. Harnett, J. Templeton, K. Dunphy-Gazman, Y. Senousy, and M. Kanouff, Lab Chip 8, 565 (2008).

[6] H. Sugioka, Phys. Rev. E 83, 025302(R) (2011).

[7] H. Sugioka, Adv. Colloid Interface Sci. 226, 44 (2015).

[8] M. Z. Bazant, M. S. Kilic, B. D. Storey, and A. Ajdari, Adv. Colloid Interface Sci. 152, 48 (2009).

[9] H. Sugioka, Phys. Rev. E 83, 056321 (2011).

[10] N. I. Gamayunov, G. I. Mantrov, and V. A. Murtsovkin, Colloid J. USSR 54, 20 (1992).

[11] A. J. Pascall and T. M. Squires, Phys. Rev. Lett. 104, 088301 (2010).
[12] B. D. Storey, L. R. Edwards, M. S. Kilic, and M. Z. Bazant, Phys. Rev. E 77, 036317 (2008).

[13] H. Sugioka, J. Phys. Soc. Jpn. 84, 094603 (2015).

[14] M. M. Gregersen, M. B. Andersen, G. Soni, C. Meinhart, and H. Bruus, Phys. Rev. E 79, 066316 (2009).

[15] S. M. Davidson, M. B. Andersen, and A. Mani, Phys. Rev. Lett. 112, 128302 (2014).

[16] H. Sugioka, Phys. Rev. E 90, 013007 (2014).

[17] J. A. Levitan, S. Devasenathipathy, V. Studer, Y. Ben, T. Thorsen, T. M. Squires, and M. Z. Bazant, Colloids Surf., A 267, 122 (2005).

[18] A. Delgado, F. Arroyo, F. González-Caballero, V. Shilov, and Y. Borkovskaya, Colloids Surf., A 140, 139 (1998).

[19] R. J. Messinger and T. M. Squires, Phys. Rev. Lett. 105, 144503 (2010).

[20] M. Z. Bazant, K. Thornton, and A. Ajdari, Phys. Rev. E 70, 021506 (2004). 